\title{
Least-Squares Approach for Harmonic and Interharmonic Analysis in Power System
}

\author{
Hong Luo and Hui Xue \\ China Agricultural University, Beijing, China, 100083 \\ xue_huicn@yahoo.com.cn
}

\begin{abstract}
A new algorithm based on least-squares method for harmonic and interharmonic analysis is proposed. The approach employs least-squares method and is based on a linear prediction relation for multiple sinusoidal signals. It is shown that the method allows for accurate harmonic and interharmonic estimation with high frequency resolution. The approach is compared with that of DFT method and prony method.
\end{abstract}

Keywords: Least square, linear prediction, interharmonic, harmonic.

\section{Introduction}

Harmonic/interharmonic measurement is an important function of a power quality analyzer widely used in monitoring the quality of power. The use of power electronic devices which increases the harmonic pollution in power system on the one hand and the widespread use of sensitive loads such as computers and microprocessor-based industrial controllers on the other hand signify the ever-increasing need for harmonic/interharmonic measurement devices. To monitor and maintain the power quality, there has been an increasing interest in devising harmonic/interharmonic measurement methods and devices over the last decade[1]-[2].

Discrete Fourier transform(DFT) has been widely used for spectral analysis of harmonics, however, DFT suffers from leakage and picket fence effect and suffers from the major problem of resolution because of some invalid assumptions(zero data or repetitive data outside the duration of observations) make in this method[3]-[4]. It is very important to develop better tools of interharmonic and harmonic estimation to avoid possible damages due to its influence.

This paper proposed a least square approach for harmonic and interharmonic analysis in power system. Least square is time-domain approach which has to do with the criterion for selecting a solution to the overdefined equations produced when more measurements than states are represented in the estimation problems[5], and has been widely used in power quality analysis. Tests show that this proposed approach can give an accurate harmonic and interharmonic estimation with high frequency resolution, the test results are compared with DFT and Prony method. 


\section{The Proposed Algorithm}

Suppose the power signal is represented

$$
x_{k}=\sum_{i=1}^{L} A_{i} \cos \left(\omega_{i} t_{k}+\phi_{i}\right)
$$

In [6], It has been shown that $x_{k}$ can be uniquely expressed as a linear combination of its previous $2 \mathrm{~L}$ samples as follow.

$$
x_{k}=-\sum_{i=1}^{L} a_{i} x_{k-i}
$$

Where $a_{i}=a_{2 L-i} \quad(\mathrm{i}=1,2 . . \mathrm{L})$;

Then (2) can be expressed as follows

$$
x_{k}=-\sum_{i=1}^{L} a_{i}\left[x_{k-i}+x_{k+i}\right]
$$

Since

$$
\begin{aligned}
& x_{k+1}+x_{k-1}=\sum_{i=1}^{L} 2 \cos \omega_{i} \Delta t A_{i} \cos \left(\omega_{i} t_{k}+\phi_{i}\right) \\
& \vdots \\
& x_{k+L}+x_{k-L}=\sum_{i=1}^{L} 2 \cos \omega_{i} L \Delta t A_{i} \cos \left(\omega_{i} t_{k}+\phi_{i}\right)
\end{aligned}
$$

From (1), (3) and (4) we obtain

$$
\sum_{i=1}^{L} a_{i} 2 \cos i \omega_{i} \Delta t=-1
$$

Equation (5) can be expressed as follows

$$
\sum_{i=1}^{L} a_{i}\left(e^{-j \omega_{i} \Delta t}+e^{j \omega_{i} \Delta t}\right)=-1
$$

Equation (6) can be expressed as follows

$$
\sum_{i=0}^{2 L} b_{i} e^{i \omega \Delta t}=0
$$

Where $b_{i}=b_{2 L-i}=a_{L-i},(\mathrm{i}=0,1, . . \mathrm{L}-1)$ and $b_{L}=1$. Then the positive phase angles of the roots of (7) are the frequencies of the sinusoidal. The parameter of $a_{i}$ can be obtained as follows. Since (3) can be expressed as following

$$
X_{k 0}=X_{k 1} S
$$


Where $X_{k 0}=\left[\begin{array}{c}x_{k} \\ \vdots \\ x_{k+M}\end{array}\right], \quad S=\left[a_{1}, \ldots . . a_{L}\right]^{T}$ and

$$
X_{k 1}=\left[\begin{array}{ccc}
x_{k-1}+x_{k+L} & & x_{k-L}+x_{k+L} \\
& \vdots & \\
x_{k+M-1}+x_{k+M+1} & & x_{k+M-L}+x_{k+M+L}
\end{array}\right],
$$

where the value of $M$ is bigger than $L$, Then $S$ can be solved using the least mean square method, that is

$$
S=X{ }_{k 1}^{-} X_{k 0},
$$

where $X_{k 1}{ }^{-}=\left(X_{k 1}^{T} X_{k 1}\right)^{-1} X_{k 1}^{T}$

After the parameter $a_{i}$ obtained, the frequencies can be obtained using (7), and we can construct a matrix, denoted as $\mathrm{E}$.

$$
E=\left[\begin{array}{cccc}
e^{j \omega_{1}} & e^{j \omega_{2}} & \cdots & e^{j \omega_{L}} \\
e^{j 2 \omega_{1}} & e^{j 2 \omega_{2}} & \cdots & e^{j 2 \omega_{L}} \\
\vdots & & & \\
e^{j N \omega_{1}} & e^{j N \omega_{2}} & \cdots & e^{j N \omega_{L}}
\end{array}\right]
$$

Notice

$$
\mathrm{EA}=\mathrm{Y}
$$

Where $A=\left[\begin{array}{llll}A_{1} e^{j \phi_{1}} & A_{2} e^{j \phi_{2}} & \cdots & A_{L} e^{j \phi_{L}}\end{array}\right]^{T}, Y=\left[\begin{array}{llll}x_{1} & x_{2} & \cdots & x_{N}\end{array}\right]^{T}$.

Then the amplitude and phase angle can be calculated again using the least mean square method again as

$$
A=\left(E^{\prime} E\right)^{-1} E^{\prime} Y
$$

\section{Simulation Resuls}

We investigate the performance of the proposed approach. Matlab is used to perform the simulations. The sample frequency is $3840 \mathrm{~Hz}$. And the data window length is 64 points, which is (1/60) second. The DFT, Prony and proposed method are compared for two cases.

Case 1: Suppose the test signal is as : \

$$
x(t)=\cos \left(2 \pi f_{1} t\right)+0.2 \cos \left(2 \pi f_{2} t\right)+0.2 \cos \left(2 \pi f_{3} t\right)
$$

Where $f_{1}=60.2 \mathrm{~Hz}, f_{2}=90.3 \mathrm{~Hz}, f_{3}=180.6 \mathrm{~Hz}$.

There is a $0.2 \mathrm{~Hz}$ frequency deviation of fundamental components, and the signal contains an $20 \%$ interharmonic with a frequency of $72 \mathrm{~Hz}$ and a $20 \% 3^{\text {rd }}$ harmonic 
component with a frequency of $180.6 \mathrm{~Hz}$. The measurement results using DFT, Prony and LMS are given in Fig.1. Fig.1 shows that DFT cannot separate the three components, Prony can separate the different frequency components but the amplitude estimation is mistaken, LMS can not only separate the different components, but also can give an accurate estimation of the amplitude as shown in Fig.1. In fact the frequency resolution of LMS can be very high, another experiment is given as follows.

Case 2: The test signal is $x(t)=\cos \left(2 \pi f_{1} t\right)+0.5 \cos \left(2 \pi f_{2} t\right)$

Where $f_{1}=60.2 \mathrm{~Hz}, f_{2}=64 \mathrm{~Hz}$.

There is a $0.2 \mathrm{~Hz}$ frequency deviation in the fundamental frequency, and a $50 \%$ $64 \mathrm{~Hz}$ interharmonic exists in the signal. The frequencies of the fundamental component and interharmonic frequency are near to each other which make it hard to separate them. The measurement results are given in Fig.2. Fig.2 shows that DFT and Prony cannot separate the two components because their frequencies are so near to each other, while the proposed LMS method can give an accurate estimation of these two components.
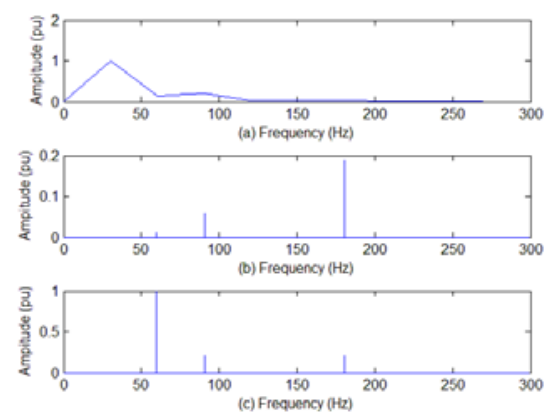

Fig. 1. Spectra obtained. (a) DFT (b) Prony (c) LMS
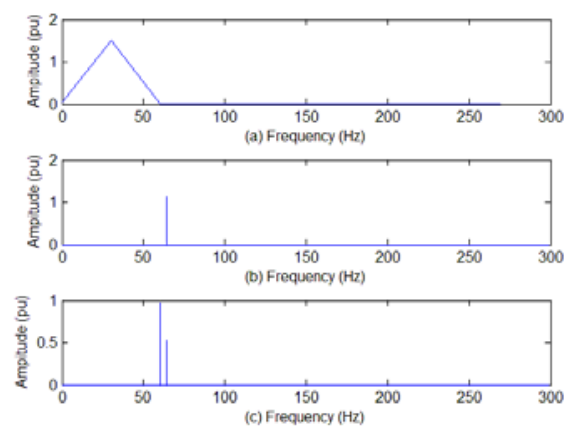

Fig. 2. Spectra obtained. (a) DFT (b) Prony (c) LMS 


\section{Conclusion}

A new algorithm based on Least-squares method for harmonic and interharmonic analysis in power system was proposed in this paper. The algorithm uses the linear prediction model of the sinusoidal. Theoretical analysis and simulation experiments validate the proposed algorithm have high frequency resolution in harmonic and interharmonic analysis.

\section{References}

1. Joorabian, M., Mortazavi, S.S., Khayyami, A.A.: Harmonic estimation in a power system using a novel hybrid least squares-adaline method. Electric Power Systems Research 79(1), 107-116 (2009)

2. Lu, Z., Ji, T.Y., Tang, W.H., Wu, H.: Optimal harmonic estimation using a particle swarm optimizer. IEEE Transactions on Power Delivery 23(2), 1166-1174 (2008)

3. Girgis, A.A., Ham, F.M.: A Quantitative Study of Pitfalls in the FFT. IEEE Transactions on Aerospace and Electronic Systems AES-16(4), 434-439 (1980)

4. Kay, S.M.: Modern Spectral Estimation: Theory and Application, pp. 224-225. PrenticeHall, Englewood Cliffs (1988)

5. Phadke, A.G., Thorp, J.S.: Synchronized phasor measurements and their applications. Springer, New York (2008)

6. Chan, Y.T., Lavoie, J.M.M., Plant, J.B.: Aparameter estimation approach to estimation of frequencies of sinusoidals. IEEE Trans. Acoust. Speech, Signal Process. ASSP 29(2), 214-219 (1981) 